\title{
Avaliação de diferentes sistemas de clareamento dental de consultório
}

\author{
Evaluation of different in-office tooth whitening systems
}

\author{
Elizandra Silva da Penha* \\ Wanessa Tomaz Pinto** \\ Rogério Lacerda dos Santos ${ }^{* * *}$ \\ Gymenna Maria Tenório Guênes ${ }^{* * *}$ \\ Luanna Abílio Diniz Melquíades de Medeiros* \\ Anny Mirene Alves de Lima**
}

\section{Resumo}

Objetivo: o objetivo deste trabalho é avaliar dois produtos de clareamento dental de consultório e constatar sua eficácia de clareamento e sensibilidade dentária pós-tratamento. Materiais e método: foram selecionados doze voluntários, a arcada superior de cada um passou por uma divisão imaginária a partir da linha mediana, formando dois grupos; o LD composto pela hemiarcada superior direita (dentes 11-15) e o LE composto pela hemiarcada superior esquerda (dentes 21 25). A arcada inferior formou grupo de controle. $O L D$ recebeu peróxido de hidrogênio a 35\% Whiteness HP® (FGM Produtos Odontológicos, Joinville, SC, Brasil) e o LE recebeu o peróxido de hidrogênio a 35\% Pola Office $^{\circledR}$ (SDI Limited, Bayswater, VIC, Austrália) em três sessões seguidas com intervalo de uma semana. A cor foi avaliada com escala visual Vitapan Classical (Vita Zahnfabrick, Bad Säckingen, BW, Alemanha) ao iniciar cada sessão e doze dias após a última sessão do tratamento clareador. Ao final de cada sessão, os voluntários registraram a ocorrência de sensibilidade dentária na escala de faces de classificação de dor de Wong-Backer. Resultados: os agentes clareadores usados apresentaram mesmo potencial clareador e não houve diferença estatística significante $(P<0,05)$ quanto à intensidade da sensibilidade quando comparados os dois produtos. Conclusão: não houve diferença entre os produtos no que diz respeito ao poder de clareamento e sensibilidade dentária.

Palavras-chave: Clareamento dental. Peróxido de hidrogênio. Pigmentação.

\section{Introdução}

A incessante busca pelo belo é preocupação não apenas na sociedade moderna, mas desde as civilizações mais antigas. Um exemplo que merece destaque é a Grécia, onde a simetria e as proporções corretas conformavam os cânones da beleza ${ }^{1}$.

Um sorriso harmônico é uma característica marcante para definir uma face como atrativa, alguns fatores como a forma, o tamanho, a cor dos dentes e o seu posicionamento nos arcos dentais estão fortemente ligados à aparência do sorriso. Alterações em alguns desses fatores podem interferir negativamente na vida profissional, ocasionar dificuldades de interação social e diminuir a autoestima do indivíduo ${ }^{2}$.

Modificações na cor dos dentes podem ser causadas por fatores extrínsecos e intrínsecos. As pigmentações extrínsecas podem ser obtidas com o consumo de alimentos com corantes, devido ao hábito de fumar e ao uso de alguns medicamentos. Essas manchas são removidas facilmente, visto que estão localizadas sobre a superfície do dente ${ }^{3-4}$.

As manchas intrínsecas são de difícil remoção, pois estão incorporadas à estrutura do dente. As pigmentações intrínsecas são classificadas como congênitas e adquiridas. As adquiridas podem ser resultantes de traumatismos dentários, tratamento endodôntico, enquanto que as congênitas são causadas por amelogênese imperfeita, fluorose, dentre outros motivos. Nesses casos, são indicados como

Professoras mestras, Departamento de Odontologia da Universidade Federal de Campina Grande, Patos, PB, Brasil.

Graduanda em Odontologia, Departamento de Odontologia da Universidade Federal de Campina Grande, Patos, PB, Brasil.

* Professor(a) doutor(a), Departamento de Odontologia da Universidade Federal de Campina Grande, Patos, PB, Brasil. 
terapêutica o clareamento dental ou restaurações diretas ou indiretas, dependendo do grau de escurecimento do dente ${ }^{5}$.

O tratamento clareador pode ser realizado nas seguintes modalidades: o clareamento no consultório, o caseiro e a associação das técnicas de consultório e caseiro. No clareamento caseiro, o paciente aplica o gel clareador com o auxílio de moldeiras personalizadas durante o tempo determinado pelo cirurgião-dentista. Essa técnica destaca-se por seu baixo custo, pela segurança no uso de agentes clareadores em baixa concentração e resultados efetivos após três a quatro semanas ${ }^{5-7}$. Dessa forma, o tratamento clareador caseiro é um procedimento eficaz, seguro e de boa procedência sob a supervisão do dentista ${ }^{8}$.

No clareamento de consultório, os agentes clareadores podem ser ativados por uma fonte de luz para acelerar o processo de branqueamento. Uma vantagem associada a essa técnica é o fato de haver menor necessidade de rigor pelo paciente (a técnica é controlada pelo profissional) e o fato de os resultados de exposição serem mais imediatos ${ }^{9-12}$.

Várias substâncias estão disponíveis no mercado para a realização do clareamento dental, que podem apresentar na sua composição peróxido de hidrogênio, peróxido de carbamida, perborato de sódio e hidroxilite. Os agentes clareadores à base de perborato de sódio são usados exclusivamente no clareamento de dentes desvitalizados. Já os clareadores que apresentam como componente o hidroxilite são usados com a finalidade de reduzir a sensibilidade dentária. O peróxido de carbamida é o agente clareador mais usado na técnica de clareamento caseira, enquanto que o peróxido de hidrogênio é a substância mais empregada na técnica de consultório ${ }^{4}$.

O clareamento dental é considerado uma técnica simples e eficaz, entretanto, é constante a discussão sobre os fatores deletérios que pode ocasionar. Dentre os principais estão a sensibilidade dentinária, alterações no conteúdo mineral resultante de um processo de desmineralização do esmalte dental e suas consequências, como alteração de rugosidade, microdureza e micromorfologia superficial, que tem sido sistematicamente estudadas na literatura científica ${ }^{13-15}$.

Assim, este trabalho teve como objetivo verificar a eficácia de dois produtos de clareamento dental de consultório. Para isso, foi formulada a hipótese nula de não existir diferenças entre os produtos no que diz respeito ao poder de clareamento e à sensibilidade dentinária.

\section{Materiais e método}

Este estudo foi submetido à aprovação do comitê de ética e pesquisa envolvendo seres humanos (CAAE 33011314400005186). Trata-se de uma pesquisa experimental, clínica, do tipo boca dividida, que foi realizada na Clínica-Escola de Odontologia da Universidade Federal de Campina Grande.
Segundo os critérios de inclusão para a pesquisa, foram selecionados voluntários em boas condições de saúde geral e bucal, com idade entre 18 e 30 anos, com os seis dentes anteriores superiores e pré-molares superiores, todos livres de cárie, que apresentavam coloração do incisivo central superior correspondente à cor A2 da escala de cor Vitapan Classical (Vita Zahnfabrik, Bad Säckingen, BW, Alemanha) ou mais escura, com a declaração de não estar grávida ou amamentando (em caso de participante do gênero feminino) e que concordaram participar da pesquisa por meio da assinatura do termo de consentimento livre e esclarecido.

Não foram selecionados os voluntários que apresentaram os dentes anteriores e pré-molares superiores com severa descoloração interna, tratamento endodôntico, restaurações extensas, lesões de cárie, fraturas, próteses, bruxismo, recessões, dentina exposta, abfração, doença periodontal, apinhamento, diastemas, mordidas abertas ou cruzadas e sensibilidade à bebida gelada. Também foram excluídos os pacientes que utilizaram substância clareadora de consultório ou caseira no último ano (não inclui pasta de dente, fio dental e enxaguante bucal clareadores), que apresentaram histórico de reação conhecida ao peróxido, que estavam tomando medicamentos ou relataram história individual ou familiar de neoplasias na região de orofaringe e adjacências.

Foram selecionados doze voluntários para participar do estudo, de acordo com os critérios de inclusão estabelecidos. Previamente ao tratamento clareador, os voluntários foram orientados a evitar alimentos com corantes, batons e bebidas ácidas. Os voluntários tiveram a arcada superior dividida a partir da linha mediana em duas hemiarcadas. Com isso, foram formados os grupos: LD, composto pela hemiarcada superior direita (dentes 11 ao 15), e LE, composto pela hemiarcada superior esquerda (dentes 21 ao 25). A arcada inferior formou o grupo controle LC.

O LD recebeu peróxido de hidrogênio a $35 \%$ Whiteness $\mathrm{HP}^{\circledR}$ (FGM Produtos Odontológicos, Joinville, SC, Brasil - Lote 041013), enquanto que o LE recebeu peróxido de hidrogênio a 35\% Pola Office $^{\circledR}$ (SDI Limeted, Bayswater, VIC, Austrália - Lote 1319772) (Quadro 1). Os voluntários receberam o tratamento clareador conforme os protocolos apresentados nos Quadros 2 e 3.

Quadro 1 - Composição dos géis clareadores

\begin{tabular}{|l|l|}
\hline \multicolumn{1}{|c|}{ Produto } & \multicolumn{1}{c|}{ Composição } \\
\hline $\begin{array}{l}\text { Pola office } \\
\text { Bayswater, VIC, Austrália) }\end{array}$ & $\begin{array}{l}\text { Peróxido de hidrogênio } \\
\text { a 35\%, água, espessante, } \\
\text { pigmento, catalizador e } \\
\text { dessensibilizante. }\end{array}$ \\
\hline $\begin{array}{l}\text { Whiteness HP }{ }^{\circledR} \text { (FGM } \\
\text { Produtos Odontológicos, } \\
\text { Joinville, SC, Brasil) }\end{array}$ & $\begin{array}{l}\text { Peróxido de hidrogênio } \\
\text { a 35\%, água, espessante, } \\
\text { corante e glicol. }\end{array}$ \\
\hline
\end{tabular}

Fonte: todos os quadros são de elaboração dos autores com base nos dados da pesquisa. 
Quadro 2 - Protocolo clínico do clareamento dental adotado no grupo $L D$

\section{Protocolo LD}

- Profilaxia com taça de borracha e pasta de pedra-pomes (Maquira Dental Products, Maringá, PR, Brasil - Lote 788312).

- Avaliação da cor com a escala Vitapan Classical (Vita Zahnfabrik, Bad Säckingen, BW, Alemanha).

- Aplicação do dessensibilizante Dessensibilize KF 2\% (FGM Produtos Odontológicos, Joinville, SC, Brasil Lote 030912) durante 10 minutos.

- Proteção dos tecidos moles com a barreira gengival fotopolimerizável Top Dam (FGM Produtos Odontológicos, Joinville, SC, Brasil - Lote 140314), seguindo as recomendações do fabricante.

- Manipulação do produto Whiteness HP ${ }^{\circledR}$ (FGM Produtos Odontológicos, Joinville, SC, Brasil - Lote 041013), seis gotas de peróxido de hidrogênio para duas medidas de espessante. Aplicação do gel clareador na face vestibular dos dentes. O produto foi mantido por 15 minutos, de acordo com as recomendações do fabricante.

- Aspiração do gel com o auxílio de sugador cirúrgico e limpeza dos dentes com gaze.

- Realização de mais duas aplicações. Ao fim da última aplicação, o gel foi aspirado e os dentes foram lavados e, posteriormente, polidos com pasta diamantada Diamond AC I (FGM, Joinvile, SC, Brasil - Lote 170812) e disco de feltro em baixa rotação.

- Aplicação de flúor neutro (Maquira Dental Products, Maringá, PR, Brasil - Lote 308414) por 4 minutos.

Quadro 3 - Protocolo clínico do clareamento dental adotado no grupo $L E$

\section{- Protocolo LE pomes (Maquira Dental Products, Maringá, PR, Brasi - Lote 788312).}

- Avaliação da cor com a escala vita (Vitapan ClassicalVita Zahnfabrik, Bad Säckingen, BW, Alemanha).

- Não foi aplicado dessensibilizante, visto que o fabricante não recomenda.

- Proteção dos tecidos moles com a barreira gengival fotopolimerizável Top Dam (FGM Produtos Odontológicos, Joinville, SC, Brasil - Lote 140314), seguindo as recomendações do fabricante.

- Manipulação do produto Pola Office ${ }^{\circledR}$ (SDI Limeted, Bayswater, Vic, Austrália - Lote 1319772), seis gotas de peróxido de hidrogênio para uma medida de espessante. Aplicação do gel clareador na face vestibular dos dentes, mantido por 8 minutos, de acordo com as recomendações do fabricante.

- Aspiração do gel com o auxílio de sugador cirúrgico e limpeza dos dentes com gaze.

- Realização de mais duas aplicações. Ao fim da última aplicação, o gel foi aspirado e os dentes foram lavados e, posteriormente, polidos com pasta diamantada Diamond AC I (FGM, Joinvile, SC, Brasil - Lote 1708120) e disco de feltro em baixa rotação.

- Aplicação de flúor neutro (Maquira Dental Products, Maringá, PR, Brasil - Lote: 308414) por 4 minutos.

Foram realizadas três sessões de clareamento dental com intervalos de uma semana entre cada uma. A mensuração da cor foi realizada pelo método subjetivo de acordo com a metodologia adotada no estudo de Bonafé et al. ${ }^{15}$ (2013), no qual a cor dos dentes dos voluntários foi verificada por dois avaliadores previamente calibrados (Kappa 0,80).

A avaliação foi sempre realizada no mesmo local, sob iluminação natural e nos mesmos horários. Foi usada a escala de cor Vitapan Classical (Vita Zahnfabrik, Bad Sackingem, BW, Alemanha), organizada em ordem de valores, atribuindo-se valores numéricos que vão do um ao dezesseis. A cor mais clara recebe a numeração um, que corresponde à cor B1 na escala, a cor mais escura recebe a numeração dezesseis, que corresponde à cor $\mathrm{C} 4$ na escala. $\mathrm{O}$ incisivo central superior foi tomado como referência para determinação da cor. As avaliações foram feitas antes e após as sessões de clareamento dental e doze dias após a última sessão. A avaliação de cor final foi realizada por dois avaliadores calibrados e cegos em relação ao produto que foi aplicado em cada lado.

Ao final de cada sessão de clareamento dental, os voluntários registraram a sensibilidade dentária de acordo com a escala de classificação de sensibilidade adotada no estudo de Wong e Backer ${ }^{16}$ (2001). Essa escala contém números que variam de zero a dez, em que zero corresponde à ausência de sintomatologia dolorosa e dez à bastante sintomatologia dolorosa.

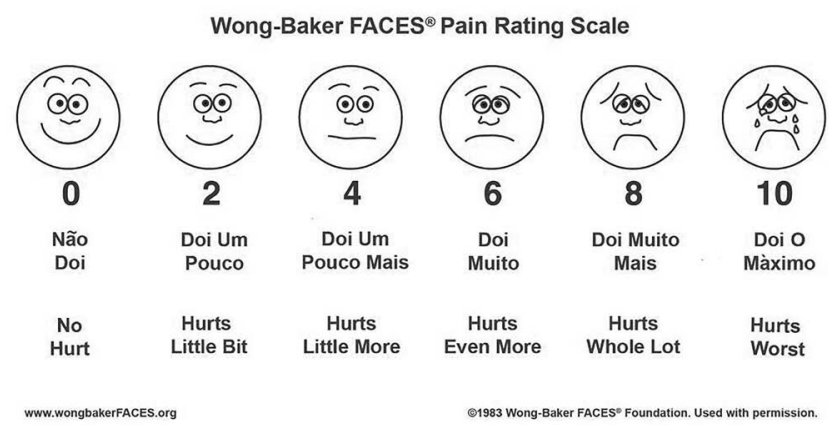

Figura 1 - Wong-Baker: escala para medir sensibilidade Fonte: WONG, 2001.

Para análise estatística da alteração da cor e da intensidade da sensibilidade dentinária ao longo do tratamento, foi utilizado o teste de Kruskal-Wallis seguido pelo teste de Dun. O teste de Mann-Whitney foi usado para comparar os dois produtos quanto à alteração de cor e à intensidade da sensibilidade dentinária.

\section{Resultados}

Dos doze pacientes selecionados, dez completaram o estudo. A aplicação dos testes de Kruskal-Wallis e de Dun mostrou que os grupos LD e LE apresentaram significante alteração de cor ( $\mathrm{P}<0.01)$. O teste de Mann-Whitney mostrou que não houve diferença estatística $(\mathrm{P}<0.05)$ em relação ao potencial clareador quando comparados os dois produtos (Tabela 1 ). 
Tabela 1 - Avaliação da cor dentária entre diferentes materiais e ao longo dos tempos avaliados

\begin{tabular}{|c|c|c|c|c|}
\hline \multirow{2}{*}{ Grupos } & Inicial & Final & $12^{\circ}$ dia pós-clareamento & \multirow{2}{*}{ P - Valor* } \\
\hline & Média (DP) & Média (DP) & Média (DP) & \\
\hline Lado D (Material 1) & $5,5(1,2)^{\mathrm{A}}$ & $1,2(0,4)^{\mathrm{B}}$ & $1,7(0,6)^{\mathrm{B}}$ & $<0,001$ \\
\hline Lado E (Material 2) & $5,5(1,2)^{\mathrm{A}}$ & $1,2(0,4)^{\mathrm{B}}$ & $1,7(0,6)^{\mathrm{B}}$ & $<0,001$ \\
\hline$P-$ Valor & 0,956 & 0,956 & 0,966 & ........... \\
\hline \multicolumn{5}{|c|}{$\overline{\mathrm{A}, \mathrm{B}} \mathrm{Em}$ linha, letras diferentes representam diferença estatística } \\
\hline \multicolumn{5}{|c|}{ * Teste de Kruskal-Wallis seguido pelo teste de Dun $(P<0.01)$} \\
\hline \multicolumn{5}{|c|}{ † Teste de Mann-Whitney $(\mathrm{P}<0.05)$} \\
\hline
\end{tabular}

A aplicação do teste de Kruskal-Wallis seguido pelo teste de Dun mostrou que entre ambos os grupos (LD e LE) não houve diferença estatística $(\mathrm{P}<0,05)$ no que diz respeito à intensidade da sensibilidade dentinária ao longo dos tempos avaliados.
O teste de Mann-Whitney mostrou que não houve diferença estatística significante $(\mathrm{P}<0,05)$ quanto à intensidade da sensibilidade dentária quando comparados os dois produtos utilizados (Tabela 2).

Tabela 2 - Sensibilidade dentária para diferentes materiais e ao longo dos tempos avaliados

\begin{tabular}{|c|c|c|c|c|}
\hline \multirow{2}{*}{ Grupos } & $1^{\mathrm{a}}$ sessão & $2^{\mathrm{a}}$ sessão & $3^{a}$ sessão & \multirow{2}{*}{ P - Valor* } \\
\hline & Média (DP) & Média (DP) & Média (DP) & \\
\hline Lado D (Material 1) & $1,7(2,4)$ & $3,6(3,5)$ & $3,9(3,1)$ & 0,321 \\
\hline Lado E (Material 2) & $1,3(2,4)$ & $2,4(2,9)$ & $3,5(2,9)$ & 0,179 \\
\hline $\mathrm{P}-$ Valor & 0,677 & 0,471 & 0,848 & ........... \\
\hline
\end{tabular}

${ }^{*}$ Teste de Kruskal-Wallis seguido pelo teste de Dun $(\mathrm{P}<0.05)$

† Teste de Mann-Whitney $(P<0.05)$

\section{Discussão}

Para este estudo foi utilizado o desenho experimental do tipo boca dividida, que permitiu a aplicação e avaliação in vivo da utilização de dois géis clareadores no mesmo paciente simultaneamente, eliminando assim possíveis variáveis que poderiam interferir nos resultados. Na literatura, os estudos clínicos sobre clareamento dentário utilizando esse tipo de desenho parecem ser escassos, e a maioria está direcionada à avaliação do uso ou não de fontes de luz durante o procedimento ${ }^{3,10,17}$.

Diversos estudos ${ }^{18}$ enfatizam que, durante o clareamento dentário, o peróxido de hidrogênio é capaz de penetrar no dente através de porosidades e trincas para então atuar diretamente sobre as moléculas do pigmento. A presente pesquisa forneceu evidência de que o princípio ativo dos géis de clareamento utilizados, peróxido de hidrogénio $35 \%$, é de fato responsável pela alteração de cor e presença de sensibilidade dentária. Os elementos inferiores (LC), que não foram submetidos à ação oxidante do peróxido de hidrogênio, não foram afetados por esses fatores.

O peróxido de hidrogênio a $35 \%$ apresenta poder clareador mesmo sendo empregado sem a utilização de fontes de luz ${ }^{19-20}$, fato confirmado pela presente pesquisa, na qual não houve emprego da fotoativa- ção do gel clareador. Entretanto, outras pesquisas afirmam que fontes de luz tornam o tratamento clareador mais eficaz e seguro ${ }^{14,17}$.

A influência dos catalizadores químicos, como os íons de ferro e manganês, aumenta a velocidade da reação por reduzir a quantidade mínima de energia cinética que as moléculas necessitam para a reação começar, proporcionando assim um caminho de menor energia ${ }^{21}$. Neste estudo, o produto empregado no LE contém na sua fórmula agentes catalizadores, e, possivelmente, devido a essa característica o grupo apresentou o mesmo potencial clareador em um menor tempo de aplicação (24 minutos por sessão) quando comparado com o produto usado no grupo LD, com tempo de 45 minutos por sessão.

Uma das divergências nas pesquisas sobre clareamento é a avaliação do efeito clareador. De acordo com critérios da American Dental Association, em 2000, a eficácia do clareamento dental pode ser avaliada por dois métodos: instrumentos de medida da cor (colorímetros ou espectrofotômetros) e escalas de cor referenciais ${ }^{22}$. Nesta pesquisa, foi utilizada para avaliação da cor dos dentes a escala universal Vitapan Classical, além de fotografias. No entanto, vale salientar que as tomadas fotográficas serviram apenas para ilustração do clareamento e não para a verificação da eficácia do procedimento. 
Alguns autores ${ }^{15,23}$ enfatizam que, embora a análise espectrofotométrica apresente-se como uma tecnologia mais precisa do que a observação visual no que diz respeito às variações das alterações de cor, a utilização da escala visual ainda é o melhor recurso clínico quando associado à habilidade e à percepção individual de cada profissional.

A sensibilidade dentinária é caracterizada por uma dor aguda, súbita e de curta duração, que acontece devido a estímulos térmicos, químicos, tácteis e evaporativos, que não pode ser referida a nenhuma outra forma de patologia dentária ${ }^{24,25}$. Frequentemente, pacientes submetidos a tratamentos clareadores queixam-se de sensações dolorosas nos dentes tratados ${ }^{26}$. Acredita-se que essas sensações dolorosas sejam decorrentes da penetração do peróxido de hidrogênio no esmalte e na dentina, que pode atingir até a polpa ${ }^{27}$.

Os agentes dessensibilizantes atuam obliterando os túbulos dentinários ou aumentando o limiar de excitação dos nervos pulpares. O fluoreto de sódio é comumente utilizado para diminuir a permeabilidade da dentina, já para ação neural emprega-se o nitrato de potássio ${ }^{28}$. Navarro et al. ${ }^{29}$ (2014), em seu estudo, constataram que o fluoreto de sódio e o nitrato de potássio diminuem a incidência de sensibilidade dentinária durante o clareamento dental ${ }^{29}$.

No presente estudo foram seguidas as recomendações dos fabricantes. Assim, foi aplicado no LD um gel dessensibilizante à base de nitrato de potássio e fluoreto de sódio anteriormente a cada sessão de branqueamento, e no LE foi aplicado um gel clareador que continha em sua fórmula agentes dessensibilizantes. Não foi possível constatar a eficácia dos dessensibilizantes presentes na composição do gel clareador nem daqueles usados antes das sessões. Esse fato pode ter sido decorrente do limitado tamanho da amostra, tornando necessárias pesquisas adicionais com número superior de voluntários para a determinação dessa característica.

Outro aspecto importante é o fato de a dor ser considerada um fenômeno multidimensional e subjetivo, que recebe influência de fatores emocionais positivos ou negativos, culturais e sensoriais. A subjetividade torna difícil a sua mensuração $0^{30}$. No presente estudo, foi avaliado o grau de sensibilidade dentária por meio da escala de faces de Wong-Backer ${ }^{16}$ (2001) ao final de cada sessão de clareamento dental. Os resultados demonstraram que a sensibilidade variou bastante, tanto entre os pacientes quanto em relação ao mesmo paciente ao decorrer das sessões, e entre o LE e o LD (hemiarcos) de cada voluntário.

$\mathrm{O}$ fato de a sensibilidade ter sido avaliada imediatamente após o clareamento dental pode ter interferido nos resultados obtidos, visto que muitos pacientes referem dor nas primeiras 24 horas após a sessão de tratamento ${ }^{15}$. Desse modo, sugere-se que mais estudos clínicos sejam realizados para analisar a sensibilidade dentária desses produtos nesses períodos.
Sabe-se que atualmente, com os diversos materiais disponíveis para o alcance da cor dental desejada, torna-se mais difícil para o cirurgião-dentista optar entre os materiais clareadores. No entanto, essa é uma decisão que cabe ao profissional, que deve avaliar as diferentes possibilidades, adequando-as à cada paciente no intuito de oferecer satisfação com o resultado, além de conforto trans e pós-operatório.

\section{Conclusão}

Foi confirmada a hipótese nula de que não existem diferenças entre os géis clareadores utilizados no que diz respeito ao poder de clareamento dentário e à sensibilidade dentária.

\section{Abstract}

Objective: To evaluate two in-office tooth whitening products and verify their effectiveness and post-treatment tooth sensitivity. Methods: 12 volunteers were selected and each of their upper arches underwent an imaginary division from the midline, forming two groups: the $L D$ group comprises the upper right hemiarch (tooth 11-15), and the LE group comprises the upper left hemiarch (tooth 21-25). The lower arch formed the control group. The LD group received 35\% hydrogen peroxide Whiteness HPTM (FGM Dental Products, Joinville, SC, Brazil) and the LE group received 35\% hydrogen peroxide Pola Office ${ }^{T M}$ (SDI Limited, Bayswater, VIC, Australia) in three consecutive sessions with a one-week interval. Color was evaluated with the visual scale Vitapan Classical (Vita Zahnfabrick, Bad Sackingen, $B W$, Germany) at the beginning of each session and 12 days after the last session of the whitening treatment. At the end of each session, the volunteers recorded the occurrence of tooth sensitivity in Wong-Baker pain faces rating scale. Results: The whitening agents used had the same whitening potential and there was no statistically significant difference $(P<0.05)$ regarding the intensity of sensitivity when comparing both products. Conclusion: There was no difference between the products regarding whitening potential and tooth sensitivity.

Keywords: Tooth whitening. Hydrogen peroxide. Pigmentation.

\section{Referências}

1. Camargos CN, Mendonça CA, Duarte SM. Da imagem visual do rosto humano: simetria, textura e padrão. Saúde e Soc 2009; 18(3):395-410.

2. Bispo LB. Clareamento dentário contemporâneo "high tec" com laser: uma revisão. Odonto Ciência 2006; 41(51):87-91.

3. Fonseca AML. Clareamento dental com a utilização do laser: um estudo controlado de boca dividida. [Dissertação de Mestrado]. Rio de Janeiro: Universidade Estácio de Sá; 2011.

4. Soares FF, Sousa JAC, Maia CC, Fontes CM, Cunha LG, Freitas AP. Clareamento em dentes vitais: uma revisão literária. Saúde.com. 2008; 4(1):72-84. 
5. Silva FM, Nacano LG, Gava Pize EC. Avaliação clínica de dois sistemas de clareamento dental. Rev Odontol Bras Cent 2012; 21(56):473-79.

6. Moura EN, Freitas LLD, Silva NCF, Sousa Júnior MHS, Loretto SC. Influência do clareamento dental fotoativado na resistência da união adesiva ao esmalte. Rev Odontol Bras Cent 2011; 20(53):140-45.

7. Know SR. Whitening the single discolored tooth. Dent Clin N Am 2011; 55:229-39.

8. Cardoso PC. Influência do tempo de aplicação de um gel clareador a base de peróxido de carbamida a $10 \%$ na cor e sensibilidade de dentes naturais. [Tese de Doutorado]. Florianópolis: Universidade Federal de Santa Catarina; 2006.

9. Marson FC, Sensi LG, Araujo FO, Andrada MACA, Araújo E. Na era do clareamento dentário a laser ainda existe espaço para o clareamento caseiro? R Dental Press Estet 2006; 3(1):89-98.

10. Zanin F, Freitas PM, Aranha ACC, Ramos TM, Ramos TM, Lopes AO. Clareamento de dentes vitais com a utilização de luz. Rev Assoc Paul Cir Dent 2010; 64(5):338-45.

11. Nunes MF, Masotti AS, Rolla JN, Soares CG, Conceição EN. Avaliação do efeito de duas técnicas de clareamento dental em consultório utilizando peróxido de hidrogênio. Rev Fac Odontol Porto Alegre 2009; 50(2):8-11.

12. Thickett E, Cobourne MT. New developments in tooth whitening. The current status of external bleaching in orthodontics. J Orthod 2009; 36:194-201.

13. Andrade AP. Monitoramento do processo de desmineralização remineralização do esmalte dental humano durante e após o clareamento dental. [Tese de Doutorado]. São Paulo: Universidade de São Paulo; 2009.

14. Araújo RM, Torres CRG, Araújo MAM. In vitro evoluation of dental bleaching effective nessusinghy bridlights activation. Odonto Ciência 2010; 25(2):159-64.

15. Bonafé ETR, Bacovis CL, Iensen S, Loguercio AD, Reis A, Kossatz S. Tooth sensitivity and efficacy of in-Office bleaching in restored teeth. J Dent 2013; 4(1):363-9.

16. Wong DL, Baker CM. Smiling faces for pain intensity scales. Pain 2001; 89(2):295-300.

17. Strobl A, Gutknecht N, Franzen R, Hilgers RD, Lampert F, Meister J. Laser-assisted in-office bleaching using a neodymium: yttrium-aluminum-garnet laser: an in vivo study. Lasers Med Sci 2010; 25:503-9.

18. Buchalla W, Attin T. External bleaching therapy with activation by heat, light or laser: a systematic review. Dent Mater 2007; 23:586-96

19. Mondelli RFL, Azevedo JFDG, Francisconi AC, Almeida CM, Shikiriama SK. Comparative clinical study of the effectiveness od diferent dental bleaching methods two year followup. J Appl Oral Sci 2011; 20(4):435-43.

20. Machado LS, Fernanda GO, Rocha EP, Santos PH, Briso ALF, Sundefeld MLMM et al. Clinical trial evolutating color change and tooth sensitivity throughout and following inoffice bleaching. Int J Periodontics Restorative Dent 2013; 33(2):209-15

21. Dannacher JJ. Catalytic bleach: Most valuable applications for smart oxidation chemistry. J Mol Catal 2006; 251-7.

22. Siew C. American Dental Association. ADA guidelines for the acceptance of tooth-whitening products. Compend Contin Educ Dent Suppl 2000; 28:S44-7.

23. Carvalho EMOF, Robazza CRC, Lage-Marques JL. Análise espectrofométrica e visual do clareamento dental interno utilizando laser e calor como fonte catalisadora. Pesqui Odontol Bras 2002; 16(4):337-42.
24. Shintome LK, Umetsubo LS, Nagayassu MP, Jorge ALC, Gonçalves SEP, Torres CRG. Avaliação clínica da laserterapia no tratamento da hipersensibilidade dentinária. Ciênc Odontol Bras. 2007; 10(1):26-36.

25. Mathias MNA, Leao JC, Menezes PF, Silva CHV. Hipersensibilidade dentinária: uma revisão de literatura. Odontol Clíncient 2010; 9(3):205-8

26. Markosvitz K. Painful: why does tooth bleaching hurt? Med hypotheses 2010; 74:835-40.

27. Ontiveros JC, Eldiwany MS, Paravina R. Clinical effectivennes and sensitivity with overnight use of $22 \%$ carbamide peroxide gel. J Dent 2012; 40:17-24.

28. Rabelo D, Loureiro M, Ferreira P, Paula A, Carrilho E. Tratamento médico dentário de hipersensibilidade dentinária: o estado da arte. Rev Port Estomatol Med Dent Cir Maxilofac 2011; 52:98-106.

29. Navarro CO, Reda B, Diolosa M, Casula I, Di Leonarda R, Breschi $\mathrm{L}$ et al. The effects of two $10 \%$ carbamide peroxide nightguard bleaching agentes, with and without desensitizer, on enamal and sensitivity: na in vivo study. Int J Dent Hyg 2014; 12(2):115-20.

30. Bottega FH, Fontana RT. A dor como quinto sinal vital: utilização da escala de avaliação por enfermeiros de um hospital geral. Texto Contexto Enferm 2010; 19(2):283-90

\section{Endereço para correspondência:}

Elizandra Silva da Penha

Universidade Federal de Campina Grande/

Ciênc. Biológicas

Avenida Universitária S/N, Santa Cecília

58708-110 Patos-PB

Telefones: (83) 3511-3000/ 9944-4500

E-mail: elizandrapenha@hotmail.com

Recebido: 19/08/15. Aceito: 30/11/15. 